\title{
cmaOPEN \\ Research
}

\section{Trends in elective and ruptured abdominal aortic aneurysm repair by practice setting in Ontario, Canada, from 2003 to 2016: a population-based time-series analysis}

\author{
Konrad Salata MD, Mohamad A. Hussain MD PhD, Charles de Mestral MD PhD, Elisa Greco MD MEd, \\ Badr A. Aljabri MD, Sandra Sabongui, Muhammad Mamdani PharmD MPH, Thomas L. Forbes MD, \\ Deepak L. Bhatt MD MPH, Subodh Verma MD PhD, Mohammed Al-Omran MD MSc
}

\section{Abstract}

Background: Recent years have seen centralization of vascular surgery services in Ontario. We sought to examine the trends in overall and approach-specific elective and ruptured abdominal aortic aneurysm repair by hospital type (teaching v. community).

Methods: We conducted a population-based time-series analysis of elective and ruptured abdominal aortic aneurysm repairs in Ontario, Canada, from 2003 to 2016. Quarterly cumulative incidences of repairs per 100000 Ontarians aged 40 years and older were calculated. We fit exponential smoothing models to the data stratified by approach and hospital type to examine repair trends.

Results: We identified 19219 elective and 2722 ruptured repairs between 2003 and 2016. The cumulative incidences of overall elective repair and elective open surgical repair decreased by $1.15 \%(p=0.008)$ and $67 \%(p<0.001)$, respectively, in teaching hospitals and by $23 \%(p<0.001)$ and $60 \%(p<0.001)$, respectively, in community hospitals. The cumulative incidence of elective endovascular repair increased $667 \%$ in teaching hospitals $(p<0.001)$. Elective endovascular repair began in community centres after 2010 and increased to $0.98 / 100000(p<0.001)$, resulting in a rebound in overall elective repair in the community. Overall ruptured repairs and ruptured open repairs decreased by $84 \%(p<0.001)$ and $88 \%(p=0.002)$, respectively, at community hospitals. Ruptured endovascular repairs at community hospitals increased from no procedures before 2006 to 0.03/100 000 in $2016(p=0.005)$.

Interpretation: There has been substantial uptake of endovascular aortic repair in teaching and community hospitals in Ontario, and community hospital uptake of endovascular repair has begun decentralization of abdominal aortic aneurysm repair. Increased experience and training in endovascular repair and reduced specialized care requirements will probably lead to continued decentralization.

\begin{abstract}
- $t$ is well known that open surgical repair of abdominal aortic aneurysm is associated with 30-day mortality as high as $7 \% .{ }^{1}$ However, studies have shown that outcomes can be improved when this surgery is conducted at experienced centres. Landon and colleagues reported that perioperative mortality at centres conducting more than 50 open repairs per year was more than $3 \%$ lower than at centres that conducted fewer than 10 per year. ${ }^{1}$ Elsewhere, larger hospital size, academic hospital type and greater annual surgeon volume have been associated with significantly lower 30-day complication and reoperation rates. ${ }^{2,3}$ Consequently, many jurisdictions have moved toward centralization of abdominal aortic aneurysm care to improve patient outcomes.

The advent of endovascular aortic repair, a minimally invasive alternative to open surgical repair, may have removed the need for centralization. Studies comparing endovascular to open abdominal aortic aneurysm repair have demonstrated superior short-term mortality and morbidity for endovascular repair, as well as shorter procedure times, lower transfusion
\end{abstract}

requirements, shorter ventilation times and reduced intensive care unit and hospital lengths of stay. ${ }^{4-7}$ However, others have demonstrated a loss of mortality benefit with long-term follow-up, higher re-intervention rates primarily for treatment of leaks, the need for lifelong surveillance to monitor for and address complications, and potential cost-effectiveness issues $^{8-13}$ (Box 1). Furthermore, work has also shown the importance of individual volume requirements for competence in endovascular repair and significant shortening of the learning curve for surgeons who learn at experienced centres. ${ }^{14,15}$ These factors suggest that even though endovascular

Competing interests: See the end of the article.

This article has been peer reviewed.

Correspondence to: Mohammed Al-Omran, alomranm@smh.ca CMAJ Open 2019. DOI:10.9778/cmajo.20180173 


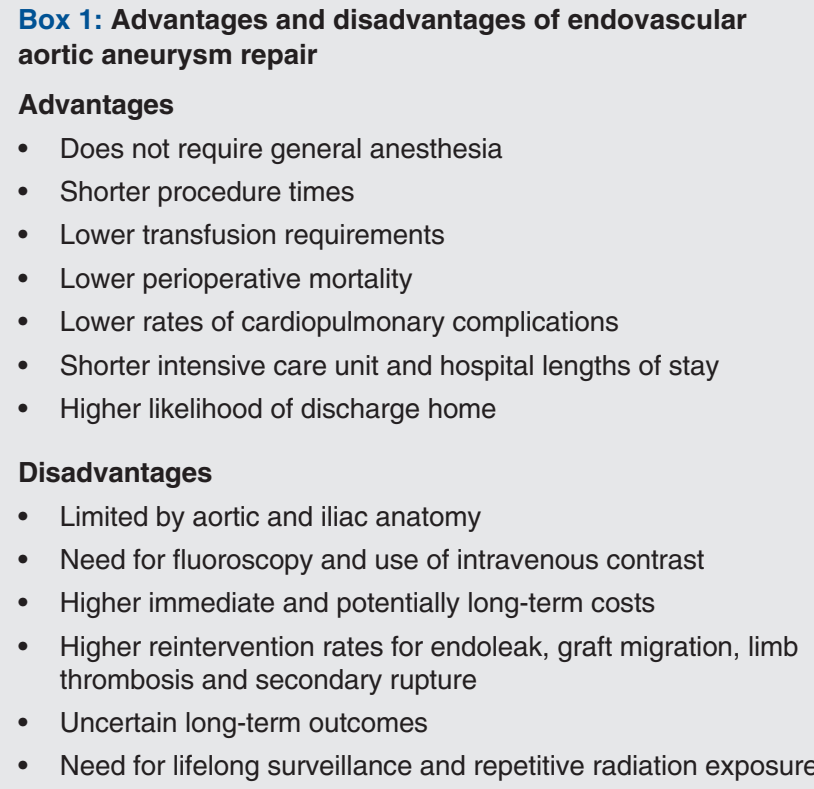

aortic repair is associated with better outcomes and fewer requirements for specialized care, abdominal aortic aneurysm repair should remain centralized at large academic hospitals. The approach-specific trends in elective and ruptured abdominal aortic aneurysm repair by practice setting have not been well studied, and the results of efforts to centralize abdominal aortic aneurysm care in the endovascular era are not clear. The purpose of the present study is to determine the trends in open and endovascular procedures for elective and ruptured abdominal aortic aneurysm repair stratified by practice setting in Ontario, Canada, from 2003 to 2016.

\section{Methods}

\section{Study design and setting}

We conducted a population-based trends analysis of open surgical and endovascular procedures for elective and ruptured abdominal aortic aneurysms repair in Ontario.

\section{Data sources}

Data for this study were obtained from ICES, a prescribed entity governed under Ontario's Personal Health Information Protection Act. ICES stores and manages data derived from multiple primary data sources that contain information on use of ambulatory, emergency and inpatient health care system interactions requiring the use of an Ontario health card. These data are anonymized and linked using a key number. The specific data sets used for this study were the Canadian Institute for Health Information Discharge Abstract Database and Same Day Surgery Database, the National Ambulatory Care Reporting System database, the Ontario Health Insurance Plan database and the Institution Information System database. ${ }^{16}$ Canadian Institute for Health Information databases have been the subject of one of the largest validation studies conducted internationally. ${ }^{17}$ This reabstraction study of approximately 14500 discharges demonstrated greater than 99\% agreement regarding demographic and admission data and very high agreement, sensitivity and specificity for procedure coding. Other validation studies have devised highly accurate definitions using the above databases for a variety of health conditions including hypertension, coronary artery disease, diabetes and stroke. ${ }^{18-27}$

\section{Patient cohort}

Our study cohort consisted of all Ontarians 40 years of age and older who underwent elective and ruptured abdominal aortic aneurysm repair in Ontario, Canada, between Apr. 1, 2003, and Mar. 31, 2016. We identified patients who underwent elective and ruptured open and endovascular repair using a combination of the Canadian version of the International Statistical Classification of Diseases and Related Health Problems, 10th Revision, the Canadian Classification of Health Interventions, and Ontario Health Insurance Plan diagnostic, procedure and billing claims codes according to our validated algorithm. ${ }^{16}$ Practice setting for each repair was identified using the teaching hospital designation from within the Institution Information System database (Appendix 1, available at www.cmajopen.ca/content/7/2/E379/suppl/DC1).

\section{Statistical analysis}

The study period was divided into 52 quarterly intervals from Apr. 1, 2003, to Mar. 31, 2016. We then generated counts of overall and approach-specific elective and ruptured repairs for each quarterly interval and calculated quarterly cumulative incidences of repair stratified by practice setting (teaching v. community hospital), using the population of Ontarians aged 40 years and older according to the 2015 Canadian census and projections, as the denominator. Time-series analyses were conducted to examine the trends of overall and approachspecific elective and ruptured repair. We fit additive and multiplicative Winters exponential smoothing models to the stratified overall and approach-specific repair data. ${ }^{28,29}$ Exponential smoothing model appropriateness was assessed using autocorrelation, and partial and inverse autocorrelation plots, as well as the Box-Ljung statistic. Model fit was evaluated using Akaike's information criterion and the adjusted $R^{2}$ values. Statistical significance was set at a 2 -sided $p$ value of 0.05 . All statistical analyses were conducted in SAS Enterprise Guide version 7.14 (SAS Institute Inc.).

We used choropleth maps to visually demonstrate changes in overall and approach-specific cumulative incidences of repair. Administrative health regions known as Local Health Integration Networks (LHINs) were used to present the data because this information is readily available and reliable within Ontario administrative data. Furthermore, the LHIN geographic subdivisions correlate well with the presence of teaching and nonteaching hospitals, with primary teaching hospitals located in 4 of the 14 LHINs (2 [South West], 4 [Hamilton-Niagara-Haldimand-Brant], 7 [Toronto Central] and 11 [Champlain]) (Appendix 1). Mean quarterly cumulative incidences of LHIN-specific repairs before 2010 and after 2010 inclusive were calculated using the Ontario population 
aged 40 years and older. The study period was divided into the aforementioned periods to demonstrate changes in cumulative incidences after the commencement of endovascular repair in community settings.

\section{Ethics approval}

This study was approved by the research ethics board at Sunnybrook Health Sciences Centre, Toronto, Ontario. The requirement for informed consent was waived because of the use of secondary data.

\section{Results}

We identified 19219 elective and 2722 ruptured repairs from Ontario administrative data. Of the patients who underwent elective repairs, 11985 (62\%) underwent open and 7234 (38\%) underwent endovascular repair. Of the patients who underwent ruptured repairs, 2458 (90\%) underwent open and 264 (10\%) underwent endovascular repairs. The mean age was 72.74 (standard deviation [SD] 8.07) years in the elective subgroup and 73.51 (SD 8.93) years in the ruptured subgroup. Most patients within each subgroup were men (15 813 [82\%] in the elective subgroup and 2178 [80\%] in the ruptured subgroup).

Approximately two-thirds of all elective repairs during the study period were conducted at teaching hospitals
(12 693/19 219), while 57\% of ruptured repairs were conducted at teaching hospitals (1562/2722). Elective and ruptured open repairs were almost equally split between teaching and community hospitals, with $56 \%$ (6724/11 985) of elective and 54\% (1336/2458) of ruptured open repairs conducted at teaching hospitals. However, 83\% (5969/7234) of elective and $86 \%$ $(226 / 264)$ of ruptured endovascular repairs were conducted at teaching hospitals.

Examination of the practice setting data over time revealed an initial increase in elective repairs at teaching centres, followed by a decrease beginning around 2010; there was a $1.15 \%$ overall reduction over the study period (from $3.62 / 100000$ in the second quarter of 2003 to 3.58/100 000 in the second quarter of $2016, p=0.008)$. In contrast, the cumulative incidence of elective repairs in community centres decreased initially then rebounded beginning around 2010 . Overall, this number decreased by $23 \%$ (from 2.61 to $2.00 / 100000, p<0.001$ ) (Figure 1 ). In teaching centres, as the cumulative incidence of elective open repair declined (from 3.30 to $1.09,67 \%$ decrease, $p<0.001$ ), the cumulative incidence of elective endovascular repair increased (from 0.34 to $2.49 / 100000,667 \%$ increase, $p<0.001)$. In contrast, community centres showed a $60 \%$ decline in the cumulative incidence of elective open repair (from 2.61 to 1.03/100 000, $p<$ $0.001)$, whereas elective endovascular repair started to

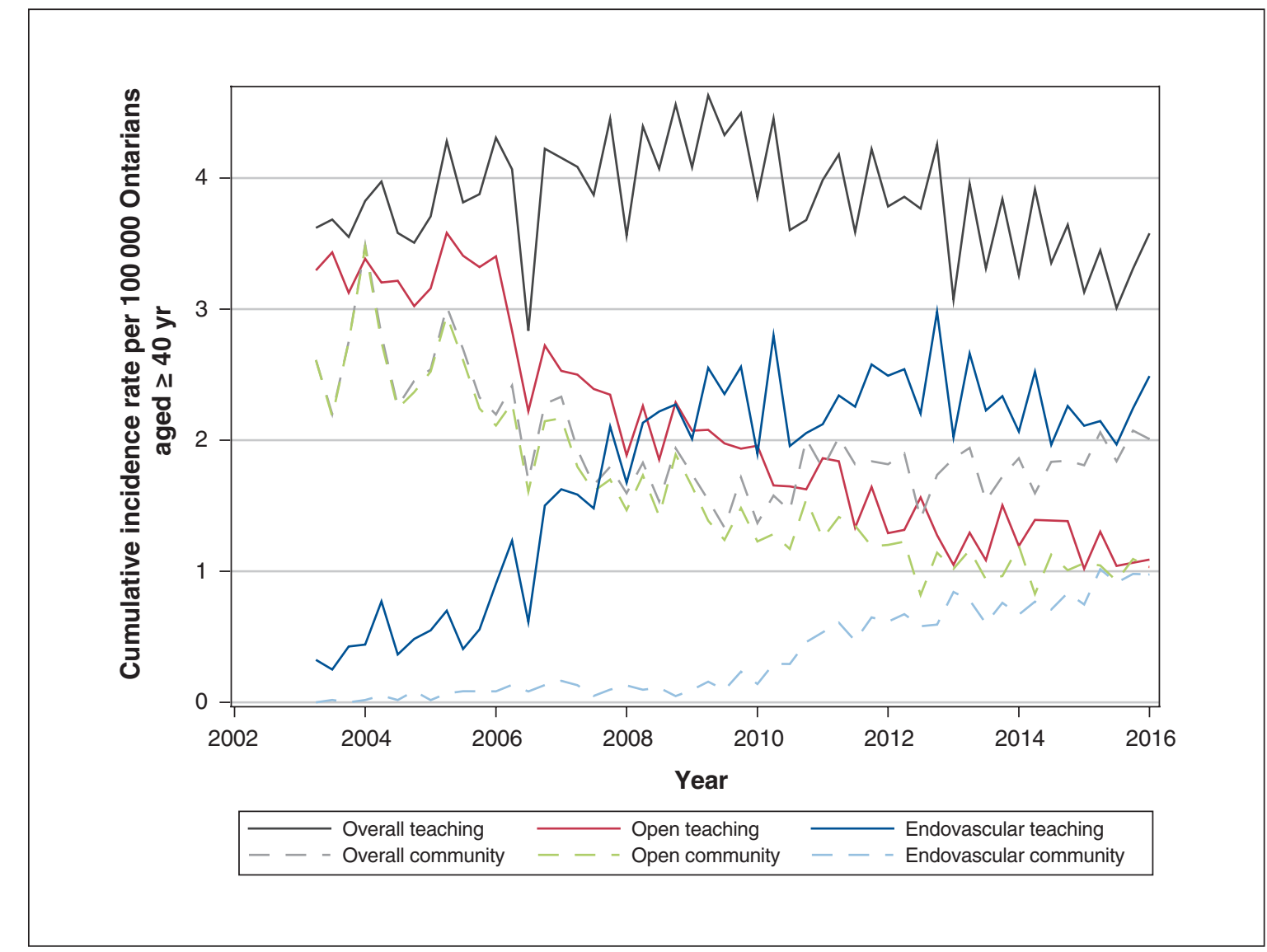

Figure 1: Actual overall and approach-specific cumulative incidences of elective abdominal aortic aneurysm repair by practice setting in Ontario from 2003 to 2016. 
increase after 2010, reaching $0.98 / 100000$ in the second quarter of $2016(p<0.001)$. Ontario choropleth maps of mean overall and approach-specific cumulative incidences of elective repair by LHIN before and after 2010 further illustrate these findings visually (Appendices 2-7, available at www. cmajopen.ca/content/7/2/E379/supp1/DC1).

There were similar trends in the overall and approachspecific cumulative incidence of ruptured repair at teaching centres, but these were not statistically significant (Figure 2). The cumulative incidence of ruptured repair decreased by $71 \%$ (from 0.90 to $0.25 / 100000, p=0.3$ ), the cumulative incidence of ruptured open repair decreased by $80 \%$ (from 0.90 to $0.18 / 100000, p=1.0$ ), and the cumulative incidence of ruptured endovascular repair increased from 0 to $0.07 / 100000$ $(p=0.1)$. Nonteaching centres saw similar but statistically significant decreases in overall ruptured repairs (from 0.72 to $0.11 / 100000,84 \%$ decrease, $p<0.001$ ) and ruptured open repairs (from 0.72 to $0.08 / 100000,88 \%$ decrease, $p=0.002$ ) and a concomitant statistically significant increase in ruptured endovascular repairs, from no procedures until late 2006 to $0.03 / 100000$ in $2016(p=0.005)$. Appendices 8-13 (available at www.cmajopen.ca/content/7/2/E379/supp1/DC1) illustrate these findings using choropleth maps of mean overall and approach-specific cumulative incidences of ruptured repair by LHIN before and after 2010.

\section{Interpretation}

Our population-based time-series analysis of overall and approach-specific elective and ruptured repairs in Ontario from 2003 to 2016, grouped by practice setting, demonstrated significant uptake of endovascular repair in teaching hospital settings and, a couple of years later, in community hospital settings. Furthermore, although cumulative incidences of endovascular repair were higher in teaching centres in both elective and ruptured contexts, the commencement of endovascular repair in community hospitals after 2010 demonstrates the beginnings of decentralization of abdominal aortic aneurysm care in Ontario.

Studies investigating the organization of aneurysm surgery services in the endovascular era are sparse. In the only Canadian study to examine the effect of endovascular repair on the organization of aneurysm surgery, Forbes and colleagues demonstrated an increase in infrarenal elective case volume from 1997 to 2003, at a tertiary teaching centre in southwestern Ontario. ${ }^{30}$ This increase was attributed to increases in referral rates from increasingly distant geographic regions and was accompanied by an increase in the proportion of these patients who received endovascular repair. Using Ontario-wide data, we confirmed the continuation of centralization of abdominal aortic aneurysm surgery to teaching centres until approximately 2010, when endovascular repair was introduced in community

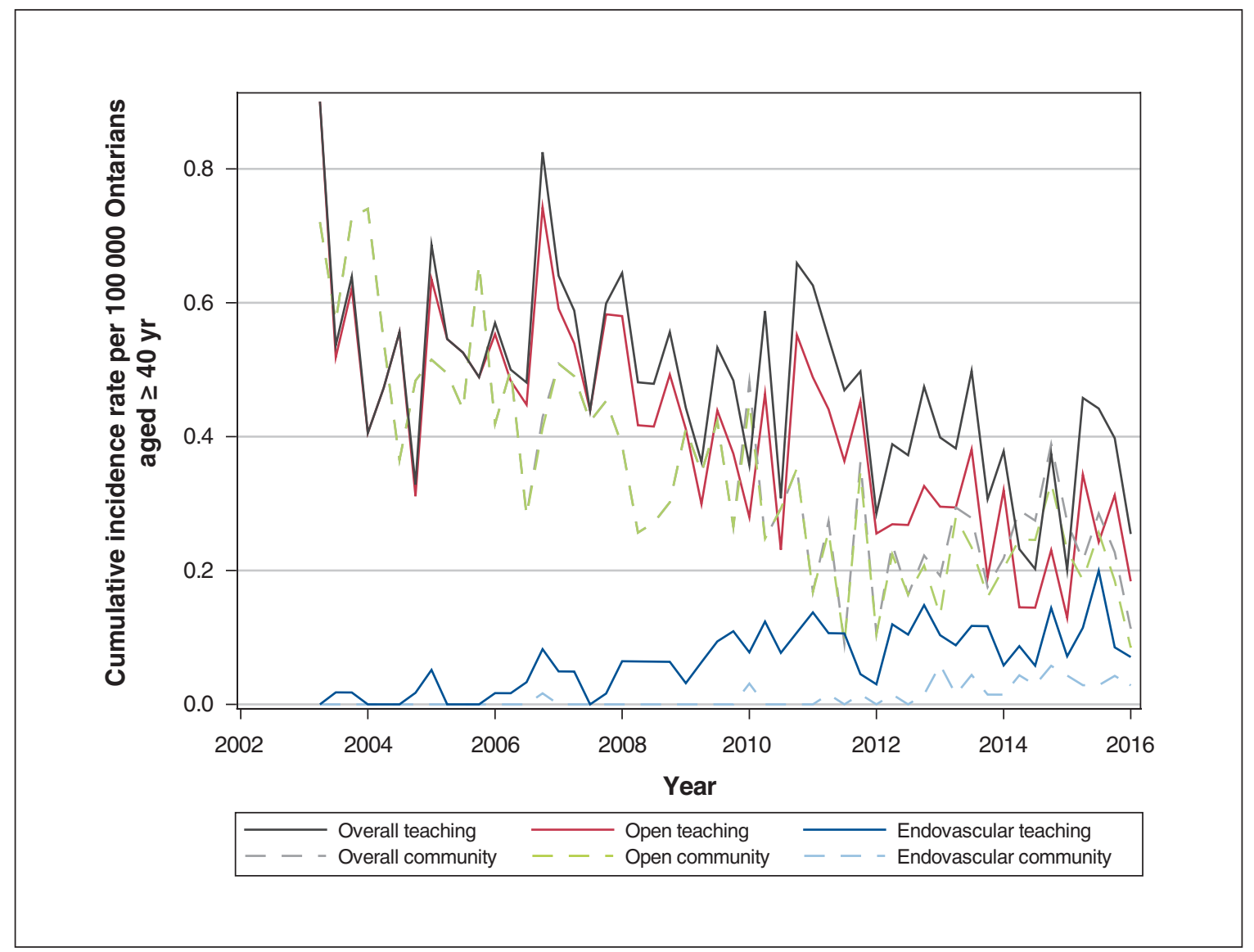

Figure 2: Actual overall and approach-specific cumulative incidences of ruptured abdominal aortic aneurysm repair by practice setting in Ontario from 2003 to 2016. 
settings and overall elective repair incidence began to recover. The well-known, substantially lower perioperative mortality risk associated with endovascular repair could have facilitated the commencement of decentralization to the community. ${ }^{4,6}$ However, the studies from which these results are derived were conducted in experienced, high-volume centres, which may not reflect the effectiveness of endovascular repair in all practice settings. Indeed, research has demonstrated volume-outcome relationships for both open and endovascular repair. In a recent population-based study, Zettervall and colleagues demonstrated lower perioperative mortality at hospitals within the highest volume quintiles of endovascular repairs ( $\geq 30$ cases per year). ${ }^{31}$ Such studies have led the Society for Vascular Surgery to recommend minimum annual volumes of 10 cases for proficiency with open and endovascular repair. ${ }^{32}$ In Ontario, funding of endovascular repair programs is conditional on more conservative volumes (30 cases per year). Increased experience, training and the minimal specialized perioperative care requirements have allowed these volumes to be achieved in community settings. Furthermore, patient preference may also have facilitated the development of these volumes. In a survey of 67 Ontarians with abdominal aortic aneurysms, Landau and colleagues found that $56 \%$ of patients would prefer surgery at a centre within a 1-hour drive of their place of residence assuming no difference in a baseline $2 \%$ perioperative mortality risk. ${ }^{33}$

The potential decentralization of endovascular repair in Ontario may have important implications for reduction of rupture. We demonstrated a decrease in the cumulative incidence of ruptured repair in both community and teaching centres. This may be related to the increasing availability of routine endovascular repair, making referral to and a wait for endovascular repair at a tertiary academic centre unnecessary, in turn reducing the incidence of rupture while patients are awaiting repair. Data on wait times from Health Quality Ontario demonstrated an average 48-day wait to see a vascular surgeon for abdominal aortic aneurysms in 2018, with an average 42-day wait until repair. ${ }^{34}$ However, these data are only available since 2015, do not encompass all Ontario hospitals and do not allow assessment of how these times have changed over the study period. Alternatively, the reductions in the incidence of rupture may be the result of endovascular repair being a management option for patients with comorbidities who are not candidates for open surgery. However, the EVAR 2 randomized trial of 388 patients physiologically unfit for open repair demonstrated no significant difference in all-cause mortality between endovascular repair and surveillance groups (hazard ratio $1.21 ; 95 \%$ confidence interval $0.87-1.69 ; p=0.3) .{ }^{35}$ The declining cumulative incidences of ruptured repair are probably related to declining rates of abdominal aortic aneurysm diagnosis, secondary to improved management of cardiovascular risk factors and reductions in smoking prevalence. ${ }^{36-38}$

\section{Limitations}

The limitations associated with population-level research should be taken into consideration when interpreting our study results. First, we used administrative codes to identify our patient cohorts. Although we validated our coding algorithms, our chart reabstraction methodology only allowed us to calculate the positive predictive values of our codes. Thus, despite confirming high positive predictive values for our codes, our study may underrepresent the number of open and endovascular repairs of elective and ruptured abdominal aortic aneurysms conducted during the study period as we were not able to calculate the sensitivity of these codes. Second, our work was conducted using Ontario administrative data. Consequently, our findings may have limited generalizability secondary to demographic and geographic factors and the presence of a single-payer publicly funded health care system. The latter may limit generalizability to jurisdictions with other health care system payment models. Similarly, demographic and geographic factors not included in our analyses may play a role in the trends of repair approach uptake. Consequently, care should be taken not to extrapolate the findings of this study outside of the scope of trend analysis.

\section{Conclusion}

Our population-based time-series analysis demonstrated significant endovascular repair uptake in both teaching and community hospitals in Ontario from 2003 to 2016. The development of endovascular repair programs at community hospitals has begun a resurgence of abdominal aortic aneurysm repair in community settings. Equivalent perioperative outcomes in community and teaching hospitals, increased experience with and training in endovascular repair, and lack of need for specialized intensive care unit teams following endovascular repair have probably contributed to this decentralization. These findings have important implications for planning for vascular surgery resource distribution in Ontario. It is likely that this shift will result in specialization of teaching centres in complex fenestrated and branched endovascular aortic repair. However, the trends in the latter types of repairs remain to be investigated.

\section{References}

1. Landon BE, O'Malley AJ, Giles K, et al. Volume-outcome relationships and abdominal aortic aneurysm repair. Circulation 2010;122:1290-7.

2. Dubois L, Allen B, Bray-Jenkyn K, et al. Higher surgeon annual volume, but not years of experience, is associated with reduced rates of postoperative complications and reoperations after open abdominal aortic aneurysm repair. 7 Vasc Surg 2018;67:1717-26.e5.

3. Hicks CW, Wick EC, Canner JK, et al. Hospital-level factors associated with mortality after endovascular and open abdominal aortic aneurysm repair. 7AMA Surg 2015;150:632-6.

4. Greenhalgh RM, Brown LC, Kwong GP, et al.; EVAR trial participants. Comparison of endovascular aneurysm repair with open repair in patients with abdominal aortic aneurysm (EVAR trial 1), 30-day operative mortality results: randomised controlled trial. Lancet 2004;364:843-8.

5. Prinssen M, Verhoeven EL, Buth J, et al.; Dutch Randomized Endovascular Aneurysm Management (DREAM) Trial Group. A randomized trial comparing conventional and endovascular repair of abdominal aortic aneurysms. $N$ Engl $\mathcal{F}$ Med 2004;351:1607-18.

6. Lederle FA, Freischlag JA, Kyriakides TC, et al.; Open Versus Endovascular Repair (OVER) Veterans Affairs Cooperative Study Group. Outcomes following endovascular vs open repair of abdominal aortic aneurysm: a randomized trial. FAMA 2009;302:1535-42.

7. Becquemin JP, Pillet JC, Lescalie F, et al.; ACE trialists. A randomized controlled trial of endovascular aneurysm repair versus open surgery for abdominal aortic aneurysms in low- to moderate-risk patients. 7 Vasc Surg 2011;53:1167-73.e1.

8. Lederle FA, Freischlag JA, Kyriakides TC, et al.; OVER Veterans Affairs Cooperative Study Group. Long-term comparison of endovascular and open repair of abdominal aortic aneurysm. N Engl F Med 2012;367:1988-97.

9. Patel R, Sweeting MJ, Powell JT, et al.; EVAR trial investigators. Endovascular versus open repair of abdominal aortic aneurysm in 15-years' follow-up of the UK endovascular aneurysm repair trial 1 (EVAR trial 1): a randomised controlled trial. Lancet 2016;388:2366-74. 
10. van Schaik TG, Yeung KK, Verhagen HJ, et al.; DREAM trial participants. Long-term survival and secondary procedures after open or endovascular repair of abdominal aortic aneurysms. 7 Vasc Surg 2017;66:1379-89.

11. Giles KA, Landon BE, Cotterill P, et al. Thirty-day mortality and late survival with reinterventions and readmissions after open and endovascular aortic aneurysm repair in Medicare beneficiaries. 7 Vasc Surg 2011;53:6-12, 3.e1.

12. Schermerhorn ML, Buck DB, O'Malley AJ, et al. Long-term outcomes of abdominal aortic aneurysm in the medicare population. $N$ Engl 7 Med 2015; 373:328-38.

13. Epstein D, Sculpher MJ, Powell JT, et al. Long-term cost-effectiveness analysis of endovascular versus open repair for abdominal aortic aneurysm based on four randomized clinical trials. Br 7 Surg 2014;101:623-31.

14. Forbes TL, DeRose G, Kribs SW, et al. Cumulative sum failure analysis of the learning curve with endovascular abdominal aortic aneurysm repair. $\mathcal{f}$ Vasc Surg 2004;39:102-8.

15. Forbes TL, DeRose G, Lawlor DK, et al. The association between a surgeon's learning curve with endovascular aortic aneurysm repair and previous institutional experience. Vasc Endovascular Surg 2007;41:14-8.

16. Salata K, Hussain MA, De Mestral C, et al. Validation of abdominal aortic aneurysm repair codes in Ontario administrative data. Clin Invest Med 2018;41:E148-55.

17. Juurlink D, Preyra C, Croxford R, et al. Canadian Institute for Health Information Discharge Abstract Database: a validation study. Toronto: Institute for Clinical and Evaluative Sciences; 2006.

18. Al-Omran M, Tu JV, Johnston $\mathrm{KW}$, et al. Outcome of revascularization procedures for peripheral arterial occlusive disease in Ontario between 1991 and 1998: a population-based study. 7 Vasc Surg 2003;38:279-88.

19. Fan J, Arruda-Olson AM, Leibson CL, et al. Billing code algorithms to identify cases of peripheral artery disease from administrative data. $7 \mathrm{Am} \mathrm{Med}$ Inform Assoc 2013;20:e349-54

20. Gershon AS, Wang C, Guan J, et al. Identifying individuals with physcian diagnosed COPD in health administrative databases. COPD 2009;6:388-94.

21. Hall R, Mondor L, Porter J, et al. Accuracy of administrative data for the coding of Acute stroke and TIAs. Can 7 Neurol Sci 2016;43:765-73.

22. Hux JE, Ivis F, Flintoft V, et al. Diabetes in Ontario: determination of prevalence and incidence using a validated administrative data algorithm. Diabetes Care 2002;25:512-6.

23. Lee DS, Stitt A, Wang X, et al. Administrative hospitalization database validation of cardiac procedure codes. Med Care 2013;51:e22-6.

24. Schultz SE, Rothwell DM, Chen Z, et al. Identifying cases of congestive heart failure from administrative data: a validation study using primary care patient records. Chronic Dis Inj Can 2013;33:160-6.

25. Tu K, Campbell NR, Chen ZL, et al. Accuracy of administrative databases in identifying patients with hypertension. Open Med 2007;1:e18-26.

26. Tu K, Mitiku T, Guo H, et al. Myocardial infarction and the validation of physician billing and hospitalization data using electronic medical records. Chronic Dis Can 2010;30:141-6.

27. Tu K, Mitiku T, Lee DS, et al. Validation of physician billing and hospitalization data to identify patients with ischemic heart disease using data from the Electronic Medical Record Administrative data Linked Database (EMRALD). Can 7 Cardiol 2010;26:e225-8.

28. Gardner ES. Exponential smoothing: the state of the art - Part II. Int 7 Forecast 2006;22:637-66. doi: 10.1016/j.ijforecast.2006.03.005.

29. Gardner ES Jr. Exponential smoothing: the state of the art. 7 Forecast 1985; 4:1-28. doi: 10.1002/for.3980040103.

30. Forbes TL, Lawlor DK, Derose G, et al. Examination of the trend in Canada toward geographic centralization of aneurysm surgery during the endovascular era. Ann Vasc Surg 2006;20:63-8.

31. Zettervall SL, Schermerhorn ML, Soden PA, et al. The effect of surgeon and hospital volume on mortality after open and endovascular repair of abdominal aortic aneurysms. 7 Vasc Surg 2017;65:626-34.

32. Chaikof EL, Dalman RL, Eskandari MK, et al. The Society for Vascular Surgery practice guidelines on the care of patients with an abdominal aortic aneurysm. 7 Vasc Surg 2018;67:2-77.e2.

33. Landau JH, Novick TV, Dubois L, et al. Determination of patient preference for location of elective abdominal aortic aneurysm surgery. Vasc Endovascular Surg 2013;47:288-93.

34. Time from decision to having surgery or procedure. Toronto: Health Quality Ontario. Available: www.hqontario.ca/System-Performance/Wait-Times-for -Surgeries-and-Procedures/Wait-Times-for-Other-Surgeries-and-Procedures/ Time-from-Decision-to-Having-Surgery-or-Procedure (accessed 2019 Apr. 1).

35. EVAR trial participants. Endovascular aneurysm repair and outcome in patients unfit for open repair of abdominal aortic aneurysm (EVAR trial 2): randomised controlled trial. Lancet 2005;365:2187-92.

36. Anjum A, Powell JT. Is the incidence of abdominal aortic aneurysm declining in the 21 st century? Mortality and hospital admissions for England \& Wales and Scotland. Eur 7 Vasc Endovasc Surg 2012;43:161-6.

37. Karthikesalingam A, Vidal-Diez A, Holt PJ, et al. Thresholds for abdominal aortic aneurysm repair in England and the United States. NEngl f Med 2016;375:2051-9.

38. Bilano V, Gilmour S, Moffiet T, et al. Global trends and projections for tobacco use, 1990-2025: an analysis of smoking indicators from the WHO Comprehensive Information Systems for Tobacco Control. Lancet 2015; 385:966-76.
Competing interests: Deepak Bhatt reports receiving grants from Abbott, Amarin, Amgen, AstraZeneca, Bristol-Myers Squibb, Chiesi, Eisai, Eli Lilly, Ethicon, Forest Laboratories, Idorsia, Ironwood, Ischemix, Medtronic, PhaseBio, Pfizer, Regeneron, Roche, Sanofi-Aventis, Synaptic and The Medicines Company, outside the submitted work. He reports receiving personal fees from the American College of Cardiology, Bayer, Belvoir Publications, Boehringer Ingelheim, the Cleveland Clinic, the Duke Clinical Research Institute, Elsevier, the Harvard Clinical Research Institute, HMP Global, the Journal of the American College of Cardiology, the Mayo Clinic, Mount Sinai School of Medicine, the Population Health Research Institute, Slack Publications, the Society of Cardiovascular Patient Care, TobeSoft and WebMD, outside the submitted work. He reports receiving reimbursement for travel expenses from the American College of Cardiology, the American Heart Association and the Society of Cardiovascular Patient Care, outside the submitted work. He was involved in unfunded research collaborations with Flowco, Fractyl, PLx Pharma, Merck, Novo Nordisk and Takeda, outside the submitted work. He served on advisory boards or committees for the American College of Cardiology, Bayer, Boehringer Ingelheim, Cardax, Medscape Cardiology, PhaseBio Regado Biosciences and the US Department of Veterans Affairs and on the board of directors for the Boston VA Research Institute and the Society of Cardiovascular Patient Care, outside the submitted work. He reports being a site co-investigator for Biotronik, Boston Scientific, St. Jude Medical and Svelte, outside the submitted work. Subodh Verma reports receiving grants from Amgen, Boehringer-Ingelheim and Bristol-Myers Squibb and personal fees from Amgen, AstraZeneca, Boehringer Ingelheim, Eli Lilly, Merck, Novartis, Novo Nordisk, Sanofi, Servier and Valeant, outside the submitted work. No other competing interests were declared.

Affiliations: Division of Vascular Surgery (Salata, Hussain, de Mestral, Greco, Aljabri, Sabongui, Al-Omran), Li Ka Shing Knowledge Institute of St. Michael's Hospital, and University of Toronto, Toronto, Ont.; Department of Surgery (Aljabri), King Saud University, Riyadh, Kingdom of Saudi Arabia; Li Ka Shing Centre for Healthcare Analytics Research and Training (Mamdani), Li Ka Shing Knowledge Institute, St. Michael's Hospital; Leslie Dan Faculty of Pharmacy (Mamdani), University of Toronto; Division of Vascular Surgery (Forbes), Peter Munk Cardiac Centre, University Health Network, and University of Toronto, Toronto, Ont.; Brigham and Women's Hospital Heart and Vascular Center and Harvard Medical School (Bhatt), Boston, Mass.; Division of Cardiac Surgery (Verma), Li Ka Shing Knowledge Institute of St. Michael's Hospital, and University of Toronto, Toronto, Ont.

Contributors: All authors were involved in the conception and design of the study. Konrad Salata was responsible for the acquisition and analysis of data, and all authors contributed to interpreting the results. Konrad Salata drafted the initial manuscript. All authors revised the manuscript for important intellectual content, approved the final version to be published and agreed to be accountable for all aspects of this work.

Funding: This work was jointly funded by a Physicians' Services Incorporated resident research grant, the Division of Vascular Surgery at St. Michael's Hospital, Toronto, Ontario, and funds from the Department of Surgery, King Saud University, Riyadh, Kingdom of Saudi Arabia. Konrad Salata was supported in part by a Canadian Institutes of Health Research Canada graduate scholarship Master's salary support award and a Goerc and Toronto Academic Vascular Specialists Surgeon Scientist Training Program scholarship.

Acknowledgements: The authors thank Cindy Fong from ICES for administrative support and Atul Sivaswamy for help with data-set definition and creation.

Disclaimer: This study was supported by ICES, which is funded by an annual grant from the Ontario Ministry of Health and Long-Term Care (MOHLTC). The opinions, results and conclusions reported in this article are those of the authors and are independent from the funding sources. No endorsement by ICES or the Ontario MOHLTC is intended or should be inferred. Parts of this material are based on data and/or information compiled and provided by the Canadian Institute for Health Information (CIHI). However, the analyses, conclusions, opinions and statements expressed in the material are those of the authors, and not necessarily those of CIHI.

Supplemental information: For reviewer comments and the original submission of this manuscript, please see www.cmajopen.ca/content/7/2/ E379/suppl/DC1. 